\title{
Acute Kidney Injury in Patients with Cirrhosis
}

\author{
Kirk B. Russ ${ }^{1}$, Todd M Stevens ${ }^{2}$ and Ashwani K. Singal*3 \\ ${ }^{1}$ Department of Internal Medicine, UAB, Birmingham, AL, USA; ${ }^{2}$ Department of Anatomic Pathology, UAB, Birmingham, AL, USA; \\ ${ }^{3}$ Division of Gastroenterology and Hepatology, UAB, Birmingham, AL, USA
}

\begin{abstract}
Acute kidney injury (AKI) occurs commonly in patients with advanced cirrhosis and negatively impacts pre- and posttransplant outcomes. Physiologic changes that occur in patients with decompensated cirrhosis with ascites, place these patients at high risk of AKI. The most common causes of AKI in cirrhosis include prerenal injury, acute tubular necrosis (ATN), and the hepatorenal syndrome (HRS), accounting for more than $80 \%$ of AKI in this population. Distinguishing between these causes is particularly important for prognostication and treatment. Treatment of Type $1 \mathrm{HRS}$ with vasoconstrictors and albumin improves short term survival and renal function in some patients while awaiting liver transplantation. Patients with HRS who fail to respond to medical therapy or those with severe renal failure of other etiology may require renal replacement therapy. Simultaneous liver kidney transplant (SLK) is needed in many of these patients to improve their post-transplant outcomes. However, the criteria to select patients who would benefit from SLK transplantation are based on consensus and lack strong evidence to support them. In this regard, novel serum and/or urinary biomarkers such as neutrophil gelatinase-associated lipocalin, interleukins- 6 and 18 , kidney injury molecule-1, fatty acid binding protein, and endothelin-1 are emerging with a potential for accurately differentiating common causes of AKI. Prospective studies are needed on the use of these biomarkers to predict accurately renal function recovery after liver transplantation alone in order to optimize personalized use of SLK.

(C) 2015 The Second Affiliated Hospital of Chongqing Medical University. Published by XIA \& HE Publishing Ltd. All rights reserved.
\end{abstract}

Keywords: Acute kidney injury; Cirrhosis; Liver transplantation; SLK.

Abbreviations: $A C E$, angiotensin-converting enzyme; AKI, acute kidney injury; ARF, acute renal failure; ATN, acute tubular necrosis; CT, computed tomography; CTP, Child-Turcotte-Pugh; ECAD, extra-corporeal albumin dialysis; eGFR, estimated GFR; ET-1, endothelin-1; FDA, Food and Drug Administration; FENa, fractional secretion of sodium; GFR, glomerular filtration rate; GI, gastrointestinal; HRS, hepatorenal syndrome; ICU, intensive care unit; IL-18, interleukin-18; L-FABP, liver-type fatty acid binding protein; LT, liver transplant; MARS, modified activating reticular system; MDRD, Modification of Diet in Renal Disease; MELD, Model for End-Stage Liver Disease; MRI, magnetic resonance imaging; NGAL, neutrophil gelatinase-associated lipocalin; NSAIDS, nonsteroidal anti-inflammatory drugs; OLT, orthotopic liver transplant; RBC, red blood cell; SBP, spontaneous bacterial peritonitis; SLK, simultaneous liver kidney; TIPS, transjugular intrahepatic portosystemic shunt; UNOS, United Network for Organ Sharing.

Received: 05 May 2015; Revised: 11 June 2015; Accepted: 12 June 2015

DOI: $10.14218 / \mathrm{JCTH} .2015 .00015$.

*Correspondence to: Ashwani K. Singal, Division of Gastroenterology and Hepatology, University of Alabama Birmingham, Birmingham, AL 35294-0012, USA. Tel: +1-205-975-9698, Fax: +1-205-975-0961, E-mail: ashwanisingal.com@ gmail.com

\section{Introduction}

Acute kidney injury (AKI) occurs frequently in patients with end-stage liver disease and cirrhosis and portends a poor prognosis. ${ }^{1,2}$ Furthermore, mortality in cirrhosis has been shown to increase progressively in association with an increase in the severity of renal dysfunction. ${ }^{2}$ AKI can be due to prerenal, intrinsic renal, and postrenal factors. Prerenal injury results from renal hypoperfusion without damage to the glomerulus or tubules. Left untreated, prerenal injury can progress to acute tubular necrosis (ATN), a type of intrinsic renal injury. Physiologic changes that occur in decompensated cirrhotics listed for orthotopic liver transplant (OLT) place these patients at high risk for AKI. Patients with cirrhosis are unique in that they can also develop hepatorenal syndrome (HRS), a type of prerenal injury that is only seen in patients with advanced liver disease. ${ }^{3}$ The increase in patients with renal dysfunction receiving transplants, combined with the potential negative effects of significant renal dysfunction on both pre- and post- transplant survival, emphasizes the importance of early identification and treatment of AKI in these patients. In this review we will analyze renal dysfunction in cirrhosis as well as its pertinence to transplant hepatology.

\section{Prevalence of AKI in cirrhosis}

The prevalence of renal dysfunction has been reported to vary from $14-50 \%$ in patients with cirrhosis. The prevalence is estimated to be approximately $50 \%$ among patients with cirrhosis and ascites and $20 \%$ of patients with advanced cirrhosis admitted to the hospital. ${ }^{4,5}$ The wide range in prevalence is likely due to different study populations and varying definitions of renal dysfunction. For example, in one retrospective study on 932 patients with cirrhosis admitted to the intensive care unit (ICU), renal dysfunction as defined by serum creatinine $>1.5 \mathrm{mg} / \mathrm{dL}$ was reported in $14 \%$ of cases. Using the same definition, in a prospective study of 206 cirrhotics (100 with sepsis), the prevalence of renal dysfunction was reported at $17 \%$ and was higher among patients with sepsis $(27 \%$ vs. $8 \%, p<0.0001) .{ }^{6}$ In another retrospective study, acute renal failure as defined by serum creatinine increase by $>50 \%$ was reported in $23 \%$ of 82 decompensated Child-Turcotte-Pugh (CTP) stage C cirrhosis patients. ${ }^{5}$ The prevalence of AKI as defined by increase in serum creatinine by $>0.9 \mathrm{mg} / \mathrm{dL}$ was reported in $25 \%$ of patients, with 93 cirrhosis patients with baseline creatinine $<1.4 \mathrm{mg} / \mathrm{dL}^{7}$ 


\section{Definition of AKI}

The use of a fixed serum creatinine threshold in patients with cirrhosis is problematic for multiple reasons. Muscle atrophy in cirrhosis decreases synthesis of creatinine. ${ }^{8}$ Renal tubular secretion of creatinine is increased in cirrhosis, and the increased volume of distribution seen in advanced cirrhosis dilutes serum creatinine measurement. ${ }^{9,10}$ Lastly, elevated bilirubin may interfere with serum creatinine assays, giving a falsely low value. ${ }^{11}$ These factors combine to result in an overestimated glomerular filtration rate (GFR) in cirrhotic patients.

An increase in serum creatinine as small as $0.3 \mathrm{mg} / \mathrm{dL}$ is meaningful and can impact patient survival and outcomes. ${ }^{9,12}$ Hence, AKI has largely replaced the terms acute renal failure (ARF), renal dysfunction, and renal insufficiency because it takes into account smaller but still clinically significant disturbances in renal function. Further, this allows for the stratification of patients into various stages of renal injury based on degree of creatinine elevation compared to baseline value. This is relevant because patient survival is not only impacted by the stage of renal dysfunction but also by the progression of renal dysfunction on follow-up. For example, in one prospective study on 192 hospitalized patients with cirrhosis, in-hospital mortality varied from $2 \%$ for AKI stage 1, $7 \%$ for stage 2 , and up to $21 \%$ for stage 3 . Furthermore, the mortality rates were $29 \%$ and $60 \%$ for stage 1 patients progressing to stage 2 and 3, respectively. Mortality increased to $19 \%$ for patients presenting in stage 2 and then progressing to stage 3 during the hospital stay. Patients with end-stage renal disease requiring dialysis had $60-71 \%$ in-hospital mortality. ${ }^{13}$

The main problem with using the well validated AKI network classification system in cirrhosis is twofold. ${ }^{9}$ Firstly, the baseline serum creatinine may not be available from the previous week. Secondly, the urine output measurements in cirrhosis patients may be unreliable, as these patients may be oliguric despite maintaining normal GFR due to sodium retention or may have increased urine output due to use of diuretics. ${ }^{9}$ To overcome these limitations, the International Club of Ascites proposed a position paper and consensus document to consider baseline serum creatinine available up to 3 months prior instead of 1 week. ${ }^{14}$ For patients without an available baseline creatinine value, the baseline is back calculated using the Modification of Diet in Renal Disease (MDRD) formula to a GFR of $75 \mathrm{~mL} / \mathrm{m}^{2}$. Secondly, the urine output requirement in the definition has been deleted (Table 1 ).

Table 1. Diagnostic criteria of renal dysfunction in cirrhosis

\begin{tabular}{ll}
\hline Diagnosis & Definition \\
\hline Acute kidney injury & $\begin{array}{l}\text { Rise in serum creatinine of } \geq 50 \% \\
\text { from baseline or a rise of serum } \\
\text { creatinine by } \geq 0.3 \mathrm{mg} / \mathrm{dL} \text { in }<48 \mathrm{~h}\end{array}$ \\
$\begin{array}{l}\text { GFR of }<60 \mathrm{~mL} / \mathrm{min} \text { for }>3 \text { months } \\
\text { chronic kidney }\end{array}$ & $\begin{array}{l}\text { Rise in serum creatinine of } \geq 50 \% \\
\text { disease }\end{array}$ \\
Acute-on-chronic baseline or a rise of serum \\
kidney disease \\
creatinine by $\geq 0.3$ mg/dL in $<48 \mathrm{~h}$ \\
in a patient with cirrhosis whose \\
GFR is $<60 \mathrm{~mL} / \mathrm{min}$ for $>3$ months \\
calculated using MDRD6 formula.
\end{tabular}

GFR, glomerular filtration rate; MDRD, modified diet in renal disease.
Based on this consensus document, AKI in cirrhosis is now defined as an increase in serum creatinine of $\geq 50 \%$ from baseline value within the last 3 months or $\geq 0.3 \mathrm{mg} / \mathrm{dL}$ $(\geq 26.4 \mu \mathrm{mol} / \mathrm{L})$ within $48 \mathrm{~h} .{ }^{14}$ The updated criteria for AKI further classify AKI into three stages, with Stage 1 defined as an increase in serum creatinine $\geq 0.3 \mathrm{mg} / \mathrm{dL}$ or $\geq 1.5-2$ fold from baseline; Stage 2 defined as an increase $\geq 2-3$ fold from baseline; and Stage 3 defined as an increase $\geq 3$ fold from baseline, serum creatinine $\geq 4.0 \mathrm{mg} / \mathrm{dL}$ with an acute increase $\geq 0.3 \mathrm{mg} / \mathrm{dL}$, or initiation of renal replacement therapy. Using this definition, about $34 \%$ of 278 patients listed for OLT at our institution developed AKI during 1 year follow-up while waiting for LT. Further, AKI was associated with increase in waitlist mortality. ${ }^{15}$

Another problem is defining chronic kidney disease and GFR in cirrhosis. The GFR can be given by various means, and most calculated GFR values are not very accurate, especially in patients with cirrhosis. Actual GFR measurements using iothalamate clearance are very accurate, but this method is not routinely available and is of limited utility in clinical practice. ${ }^{16}$ It has been shown that the MDRD- 6 formula is the most accurate for estimating GFR in patients with cirrhosis. This is the same as the commonly used MDRD-4 formula, but it also incorporates blood urea nitrogen and serum albumin in addition to the components used for MDRD-4, which include age, gender, race, and serum creatinine value. ${ }^{17}$ It should be noted that the MDRD- 6 formula tends to underestimate GFR in a subgroup of patients whose true GFR is $>30 \mathrm{~mL} / \mathrm{min} / 1.73 \mathrm{~m}^{2}$, which may result in unnecessary simultaneous liver kidney (SLK) transplants. Factors associated with discordance in this subgroup with underestimated GFR include older age, low serum sodium, and refractory ascites. ${ }^{17}$

\section{Pathophysiology of renal dysfunction in cirrhosis}

Patients with cirrhosis develop portal hypertension with resultant splanchnic vasodilation and pooling of blood secondary to increased resistance to portal flow (Fig. 1). This is due to fixed resistance from hepatic fibrosis and dynamic resistance in splanchnic arteries due to a) vasodilators such as nitric oxide, carbon monoxide, and endogenous cannabinoids ${ }^{18,19}$ and $b$ ) vasodilation from inflammatory cytokines such as tumor necrosis factor-alpha and interleukin-6 induced by bacterial translocation from the gut. ${ }^{20}$ Pooling of blood in the splanchnic system leads to decreased effective circulatory blood volume in patients with cirrhosis. ${ }^{3}$ The compensatory increase in cardiac output via activation of the sympathetic nervous system by carotid baroreceptors maintains sufficient renal perfusion. However, with decompensation of cirrhosis and increasing severity of portal hypertension, the compensatory increase in cardiac output is inadequate to maintain circulatory blood volume and adequate renal perfusion. ${ }^{3}$ Further, the development of cirrhotic cardiomyopathy in up to $40-50 \%$ of patients with cirrhosis adds to this problem. ${ }^{21,22}$ As the severity of liver disease progresses, decreased renal perfusion causes activation of the reninangiotensin-aldosterone system, resulting in sodium and water retention and extra-splanchnic vasoconstriction. ${ }^{3,23}$ This leads to ascites and decreased renal perfusion respectively, and explains the common observation in clinical practice of AKI in cirrhosis with ascites. In one study, presence of ascites was associated with a five-fold increase in risk of AKI. ${ }^{7}$ In the setting of this systemic and renal vasoconstriction seen in advanced cirrhosis, ${ }^{24}$ prostaglandins secreted locally 
Russ K.B. et al: AKI in cirrhosis

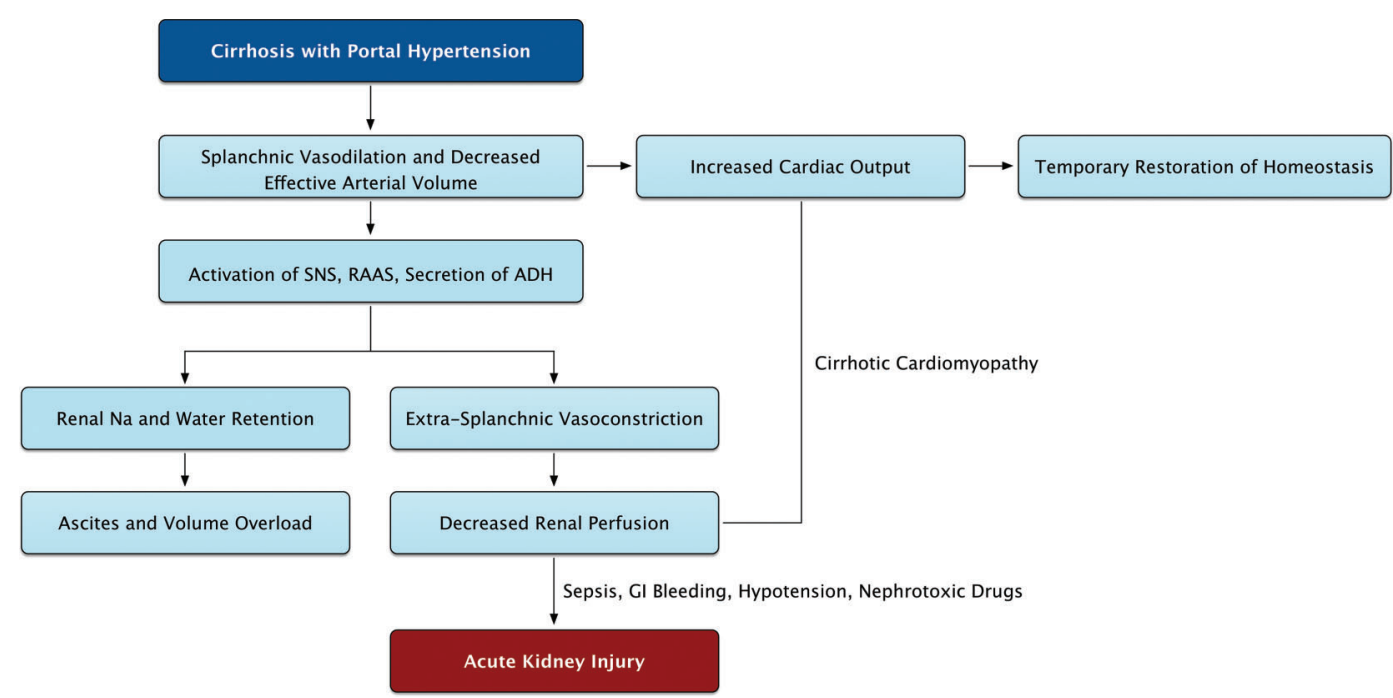

Fig. 1. The pathophysiology of renal dysfunction in decompensated cirrhosis. See text for further discussion. SNS, sympathetic nervous system; RAAS, ReninAngiotensin-Aldosterone System; ADH, antidiuretic hormone.

within the kidneys are the last compensatory mechanism for maintaining renal blood flow. ${ }^{25}$ These physiologic changes combine to set the stage for development of renal insufficiency, either spontaneously with the worsening of liver disease or precipitated by events that further worsen this fragile physiology. Common precipitants of AKI in patients with cirrhosis are use of diuretics, gastrointestinal (GI) bleeding, large volume paracentesis without albumin replacement, infections, and use of nephrotoxic drugs, including nonsteroidal anti-inflammatory drugs (NSAIDS). ${ }^{3,25}$

\section{Causes of AKI in cirrhosis}

AKI can occur due to prerenal, intrarenal, or postrenal causes. ${ }^{3,26}$ Of these, prerenal etiology is the most common cause of AKI among patients with cirrhosis followed by ATN, while the postrenal etiology due to urinary tract obstruction is extremely rare (Fig. 2). In a retrospective study of 423 patients with cirrhosis admitted to the hospital with a diagnosis of AKI, prerenal and ATN accounted for over $80 \%$ of cases (49\% prerenal; $35 \%$ ATN). ${ }^{27}$ Postrenal injury accounted for

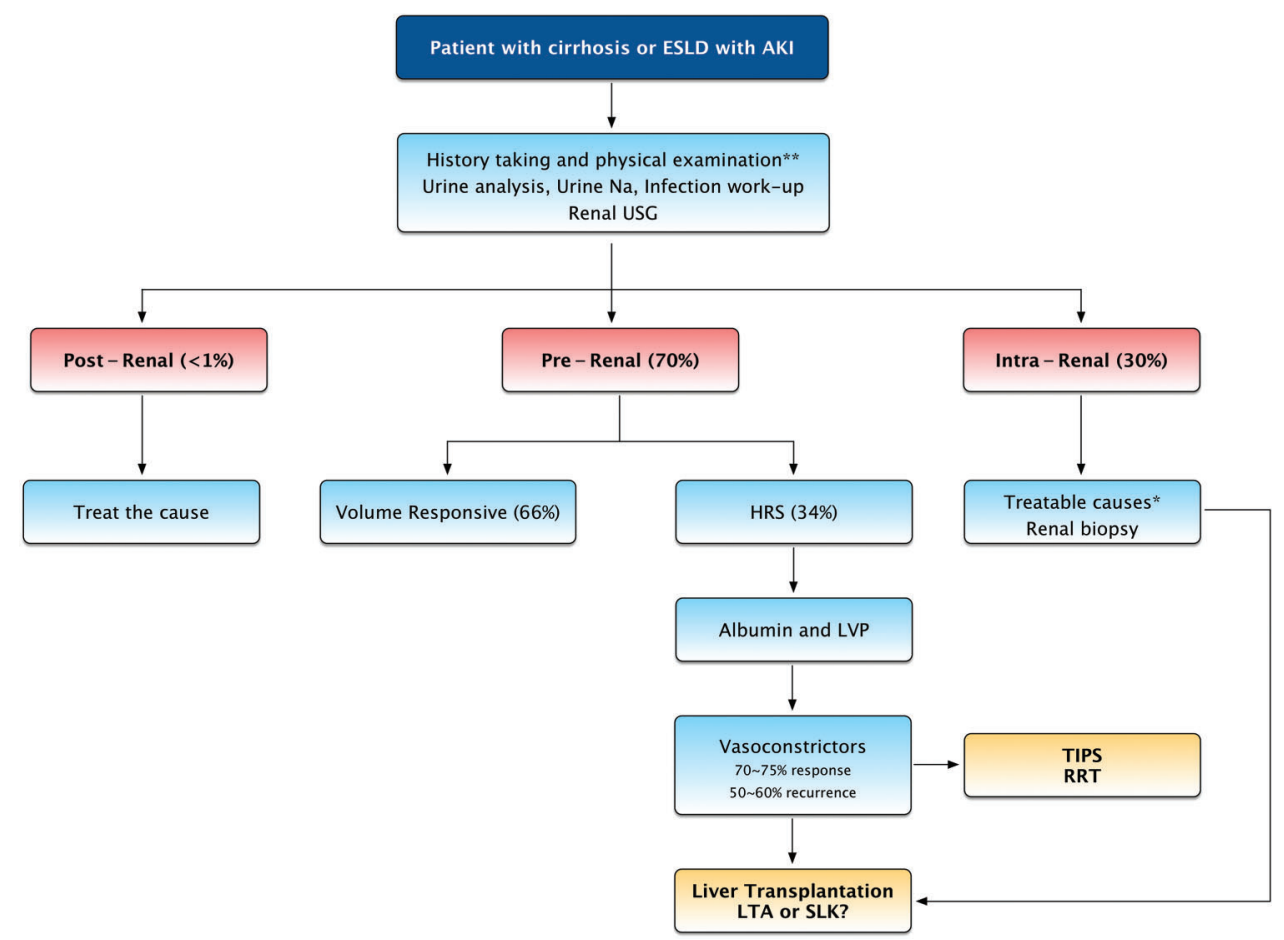

Fig. 2. Management approach and algorithm for acute kidney injury in patients with cirrhosis. ESLD, end-stage liver disease; AKI, acute kidney injury; USG, ultrasonogram; LVP, large volume paracentesis; HRS, hepatorenal syndrome; TIPS, transjugular intrahepatic portosystemic shunt; RRT, renal replacement therapy; LTA, liver transplant alone; SLK, simultaneous liver kidney. 
only $0.2 \%$ of cases in this study. ${ }^{27}$ In our prospective study, among 109 patients with cirrhosis listed for liver transplantation who had AKI, prerenal injury was the most common cause in 76 followed by intrarenal etiology in 33 , while postrenal etiology did not occur in any patient. ${ }^{15}$

\section{Prerenal injury}

Volume responsive pre-renal AKI

Due to altered hemodynamics in cirrhosis patients, these patients remain prone to AKI due to slight fluid shifts and additional decreases in intravascular fluid volume. Prerenal injury occurs commonly due to use of diuretics, GI bleeding, infection, and diarrhea often related to lactulose use for hepatic encephalopathy. ${ }^{3,26}$ Fluid losses may be further accentuated by poor oral intake from nausea/vomiting and from large volume paracentesis without albumin infusion. Large volume paracentesis may be associated with intravascular volume depletion and AKI, so-called "postparacentesis syndrome". This condition occurs in up to $70 \%$ of patients undergoing paracentesis when more than $5 \mathrm{~L}$ are removed, and albumin is not infused. ${ }^{3,26}$ Prior to the widespread use of antibiotic prophylaxis for acute GI bleeding in cirrhosis, up to $20 \%$ of patients with cirrhosis hospitalized for acute GI bleeding had a bacterial infection present on admission, with up to $50 \%$ developing an infection while hospitalized. ${ }^{28}$ Additionally, use of drugs such as NSAIDS can precipitate AKI by decreasing renal prostaglandins and accentuating the intrarenal vasoconstriction and further decrease renal blood flow. ${ }^{25}$ Hence, routine advice should be provided to these patients to avoid these drugs for management of pain whenever analgesic agents are needed. Use of intravenous contrast agents in patients with cirrhosis is another potential risk factor for AKI. ${ }^{3,26}$ In this regard, ultrasound should be the imaging modality of choice to follow patients with cirrhosis for hepatocellular carcinoma surveillance and screening. However, a suspicious lesion on ultrasound should be followed with contrast computed tomography (CT) or magnetic resonance imaging (MRI) scan to confirm or refute the diagnosis of hepatocellular carcinoma. ${ }^{29} \mathrm{~A}$ common and frequent occurrence in patients with cirrhosis is development of infections and/or sepsis. ${ }^{30}$ The hemodynamic state in cirrhosis with vascular dilatation and reduced vascular resistance in cirrhosis is quite similar to hemodynamic state in sepsis, especially spontaneous bacterial peritonitis (SBP). Hence, superimposed infections/sepsis in cirrhosis patients worsen this physiology, causing a reduction of circulating blood volume and leading to the development of AKI. 3,26,30

Volume nonresponsive prerenal AKI: hepatorenal syndrome

The first step after AKI is diagnosed is volume expansion (with crystalloids or intravenous albumin) and discontinuation of precipitating medications (such as diuretics, lactulose, and NSAIDs). If renal function does not normalize or improve with this intervention, HRS is an important differential diagnosis to consider as the cause for AKI. ${ }^{3,26}$ HRS is a functional form of renal failure without any major structural or histological changes in the kidneys that is characterized by intense renal vasoconstriction (Fig. 3 and Fig. 4). ${ }^{31}$ Since HRS has been recognized, there remains an unmet need for an accurate biomarker for diagnosis of HRS. It is important to differentiate HRS from intrarenal causes such as ATN, because

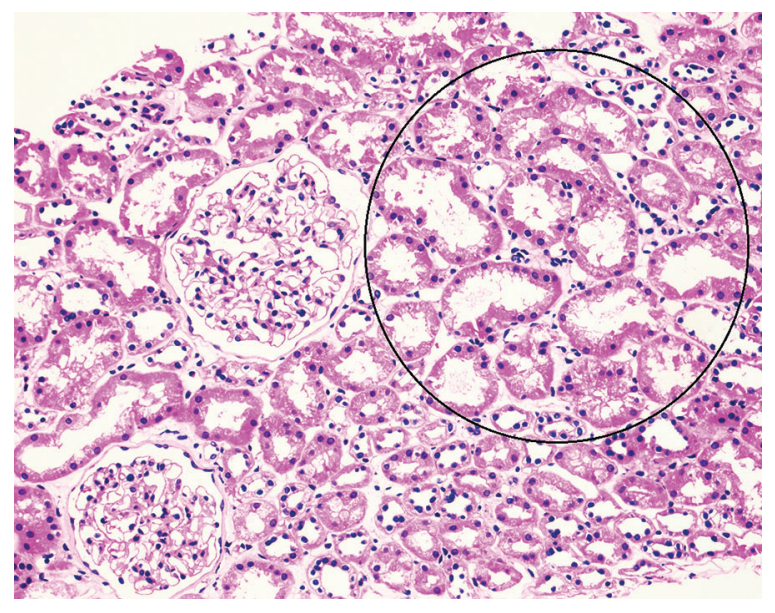

Fig. 3. Renal biopsy findings in hepatorenal syndrome. Renal biopsy findings in hepatorenal syndrome often reveal acute tubular injury with no glomerular abnormalities, as shown here. Here we see findings of acute tubular injury consisting of thinning of proximal tubular epithelial cells with widening of tubular lumens (circled area). Note the two normal glomeruli (hematoxylin and eosin stain, $200 \times)$.

management and prognosis differ. In the absence of renal biopsy, the diagnosis of HRS remains difficult and is essentially a diagnosis of exclusion (Table 2) ${ }^{14,31}$ Due to concurrent coagulopathy and thrombocytopenia in advanced cirrhosis, renal biopsy carries a high risk of internal bleeding and is rarely performed. In one study on 55 patients with cirrhosis undergoing transjugular renal biopsy, eight patients developed internal bleeding, and four had perinephric hematoma. ${ }^{32}$ In another study on 44 patients undergoing percutaneous renal biopsy, bleeding complications occurred in about one-third of the patients. ${ }^{33}$ In another study of 20 patients (five transjugular and 15 percutaneous biopsies), bleeding complication occurred in two patients. ${ }^{34}$ In our prospective study on 278 patients with cirrhosis listed for liver transplantation, none of the 109 patients who developed AKI underwent renal biopsy for evaluation of AKI. ${ }^{15}$

Approximately $75 \%$ of all HRS cases are type 1 , which are rapidly occurring with an increase in serum creatinine to over

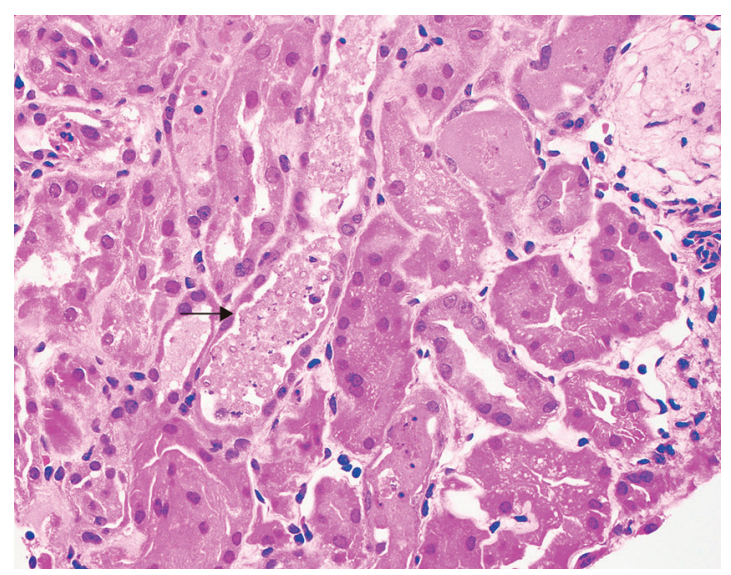

Fig. 4. Renal biopsy findings in acute tubular necrosis. In contrast, the histologic findings in acute tubular necrosis show necrotic and sloughed off tubule epithelial cells filling tubular lumens (arrow) (hematoxylin and eosin stain, 400×). 
Table 2. Diagnostic criteria for hepatorenal syndrome

- Presence of cirrhosis and ascites
- Diagnosis of AKI by ICA-AKI Criteria
- No improvement of serum creatinine after at least $48 \mathrm{~h}$ of
diuretic withdrawal and volume expansion with albumin
(recommended dose of $1 \mathrm{~g} / \mathrm{kg}$ body weight per day to
maximum of $100 \mathrm{~g}$ albumin/day
- Absence of shock
- No current or recent use of nephrotoxic drugs
- No macroscopic signs of structural kidney disease as
defined as absence of proteinuria $>500$ mg/day,
microhematuria (>50 RBCs/high power field), and/or
abnormal renal ultrasound scanning.

AKI, acute kidney injury; RBCs, red blood cells.

$2.5 \mathrm{mg} / \mathrm{dL}$ over $1-2$ weeks. Type 1 HRS is usually precipitated by infections/sepsis and has high mortality with a median survival of around $50 \%$ at 2 weeks. ${ }^{35}$ In contrast, type II HRS is more indolent in the setting of refractory ascites, with slowly increasing serum creatinine to over $1.5 \mathrm{mg} / \mathrm{dL}$ and a relatively better outcome with a median survival of about 6 months..$^{3,14,26}$ To avoid delaying the initiation of therapy, the definition of type I HRS has been recently revised to remove the ceiling of $2.5 \mathrm{mg} / \mathrm{dL}$ serum creatinine. ${ }^{14}$ In patients with cirrhosis, HRS develops in about $18 \%$ at 1 year and $39 \%$ at 5 years. ${ }^{36}$ In a retrospective study on 423 patients with cirrhosis and AKI, HRS type 1 contributed to AKI in $17 \%$ of patients. ${ }^{27}$ In another prospective study on 263 patients with cirrhosis and ascites, HRS accounted for AKI in approximately $8 \%$ of patients over a median follow-up of about 41 months after onset of ascites. ${ }^{4}$ The incidence of HRS increases with disease severity. In one study on patients listed for liver transplant, HRS was the etiology of AKI in about $48 \%$ of patients with AKI requiring renal replacement therapy. ${ }^{37}$ In our prospective study on 278 patients listed for liver transplantation, 109 had AKI over a period of one year; and HRS accounted for AKI in about $19 \%$ of these cases (type I was about $14 \%)^{15}$

\section{Volume nonresponsive intrinsic AKI: ATN}

The most common cause for intrarenal AKI in cirrhosis is ATN. This occurs commonly either as a complication of sepsis or due to unrecognized and untreated pre-renal injury. ${ }^{3,26}$ In a large retrospective report, the cause of ATN was attributed to sepsis in $61 \%$ of cases, hypovolemia in $36 \%$ of cases, and nephrotoxic drugs (aminoglycosides) in $2 \%$ of cases. ${ }^{38}$ Other less common causes of intrarenal injury include membranoproliferative glomerulonephritis with or without cryoglobulinemia associated with hepatitis $\mathrm{C}$, tubular damage due to bile cast nephropathy from high conjugated bilirubin excreted through the glomeruli, and acute interstitial nephritis due to medications, such as antibiotics, NSAIDS, and proton pump inhibitors. ${ }^{3,26,38,39}$ When the urine examination shows a high suspicion for glomerular etiology with findings of red blood cells (RBCs) and/or RBC casts, renal biopsy is often performed for diagnosing the exact etiology and treating with specific drugs.

\section{Postrenal injury}

As stated earlier, postrenal injury is a rare cause of AKI in cirrhosis. ${ }^{27}$ This etiology can easily be excluded using renal ultrasound or CT scan.

\section{Determining the cause of AKI}

As AKI significantly impacts the outcome of patients with cirrhosis, it is important to prevent the development of AKI if possible and to identify the cause early enough so that appropriate treatment measures can be instituted. ${ }^{3,14,26} \mathrm{~A}$ thorough history and careful physical examination are important to evaluate for potentially reversible and/or treatable causes for AKI, such as hypovolemia; hypotension; medications such as diuretics, lactulose, and NSAIDS; skin findings of cellulitis or line infection; and ongoing GI losses, such as nausea, vomiting or diarrhea. The threshold should be low for underlying infection as a cause of AKI, particularly SBP. Appropriate work up for infections should be performed by obtaining pan-cultures and chest radiography. Urinalysis and urine microscopy should be obtained to examine for urine sediment and detect findings suggestive of intrarenal injury such as tubular casts of ATN, microscopic hematuria, and RBC casts in glomerulonephritis or proteinuria. ${ }^{3,14,26}$ Although fractional secretion of sodium (FENa) is often utilized to help distinguish between prerenal injury and ATN in patients without cirrhosis, it is of limited utility in patients with cirrhosis. A study of 44 patients with biopsy proven ATN and cirrhosis by Wadei et al. revealed that $92 \%$ had a FENa $<1 \%{ }^{33}$ FENa may also be less than $1 \%$ in ATN from contrast induced nephropathy and sepsis. Renal ultrasound is indicated to rule out postrenal injury and obstructive disorder of the urinary tract (Fig. 2).

Volume expansion with approximately 1.5 liters of normal saline or $1 \mathrm{gm} / \mathrm{kg}$ of albumin (maximum of $100 \mathrm{gm} / \mathrm{d}$ ) should be administered as soon as AKI is identified in a cirrhosis patient. Rapid reversal of AKI over about 48 hours with bland urine sediment is suggestive of prerenal AKI. However, if the initial measures mentioned above do not reverse the AKI, it is important to determine whether the patient has HRS or ATN. ${ }^{3,26}$ Making this distinction is important for prognostication and determining which patients benefit from liver transplant alone versus combined liver kidney transplant. Routine clinical examination and urinalysis are unable to make this distinction in about $40-50 \%$ of cases. ${ }^{14,26}$ Renal biopsy, the gold standard for differentiating these two causes of AKI, is a high risk procedure and is rarely performed due to the risk of bleeding complications, as previously discussed. ${ }^{32-34}$ The diagnosis of HRS, therefore, mainly remains one of exclusion based on the recommended criteria (Table 2). ${ }^{14}$ Recently, data have emerged regarding the accuracy of urinary and/or serum biomarkers, such as neutrophil gelatinase-associated lipocalin (NGAL), interleukin-18 (IL-18), kidney injury molecule-1 (KIM-1), and liver-type fatty acid binding protein (L-FABP), to distinguish ATN from HRS. ${ }^{13,40}$ These biomarkers are promising and may prove helpful in distinguishing ATN from HRS type I, as they are more elevated in AKI due to ATN than HRS or prerenal causes of AKI. ${ }^{13}$

A prospective cohort study of 241 patients with cirrhosis examining the use of urinary NGAL as a marker for tubular injury found that urinary NGAL levels were markedly higher in patients with ATN than those patients with prerenal azotemia, chronic kidney disease, and HRS. Patients with HRS had 
intermediate urinary NGAL levels that were significantly higher than in the group of patients with prerenal AKI and significantly lower than the group of patients with ATN ${ }^{41}$ In another prospective study of 168 patients with cirrhosis and severe sepsis who developed acute renal failure, patients with ATN had significantly higher levels of urinary IL-18 compared to patients without ATN, and IL-18 was found to be an independent predictor of mortality in these patients. ${ }^{42}$ Another prospective cohort study of 188 patients with cirrhosis and AKI examined the use of NGAL, IL-18, KIM-1, L-FABP, and urinary albumin to distinguish structural from functional causes of AKI in this population. This study found that the levels of these urinary biomarkers were significantly elevated in patients with ATN compared to patients without ATN. When the biomarkers were combined, the relative risk of being diagnosed with ATN increased in a stepwise fashion for every additional biomarker that was positive. ${ }^{43}$

These biomarkers are helpful at distinguishing ATN from nonATN causes of renal failure. However, there remains an unmet need for an accurate biomarker for the diagnosis of HRS. One study found that the levels of endothelin-1 (ET-1), a molecule secreted by vascular endothelial cells, were accurate in diagnosing HRS as etiology of renal dysfunction among patients receiving liver transplantation. ${ }^{44}$ Cystatin $C$ was also found to be an independent predictor of HRS in patients with cirrhosis and ascites with normal renal function, which could prove useful for identifying patients at risk for HRS. ${ }^{45,46}$

\section{Prevention}

Since renal function impacts outcomes both before and after transplantation, prevention of AKI is crucial while managing patients with cirrhosis. Physicians caring for patients with cirrhosis should always advise patients to maintain adequate hydration and avoid nephrotoxic medications. Specifically, NSAIDS, aminoglycoside antibiotics, and angiotensin-converting enzyme (ACE) inhibitors should be avoided in these patients if at all possible. ${ }^{3,26}$ Diuretics should be used judiciously, and the doses should be optimized with careful monitoring of renal function. Patients needing paracentesis should have albumin replacement if more than $5 \mathrm{~L}$ are removed in order to avoid postparacentesis hypovolemia and precipitation of AKI. Contrast imaging should be performed only when needed, such as to confirm a suspicious lesion on ultrasound and to exclude hepatocellular carcinoma. High risk patients requiring contrasted studies should have measures instituted to prevent contrast induced nephropathy. ${ }^{47}$ Gadolinium containing MRI contrast agents should be avoided due to the risk of nephrogenic systemic fibrosis among patients with cirrhosis with renal dysfunction. ${ }^{48}$ Cirrhosis is an immune compromised state, and these patients are at increased risk of infection. ${ }^{49}$ Therefore, early evaluation and treatment of infection is imperative for patients with cirrhosis who develop an AKI. ${ }^{6,38,50}$ Patients with GI bleeding remain at risk for infections, especially SBP. Hence, third generation cephalosporins have been shown to improve survival in this population and should be used prophylactically to prevent infections. ${ }^{28}$ Patients with SBP, especially those with CTP stage B or C cirrhosis, should receive albumin infusion of $1.5 \mathrm{gm} / \mathrm{kg}$ body weight on day 1 and $1 \mathrm{gm} / \mathrm{kg}$ on day 3 , to reduce the risk of HRS ( $33 \%$ vs. $10 \%)$, along with antibiotic administration. This practice has been shown to improve survival and reduce in-hospital mortality compared to patients receiving placebo $(29 \%$ vs. $10 \%, p=0.01) .{ }^{51}$ Recently, data have emerged that albumin administration is beneficial in improving survival and prevention of AKI among patients with cirrhosis and infections other than SBP. ${ }^{52}$ Patients with an established episode of SBP are at risk of recurrence of another episode, with a rate as high as $70 \%$ over 1 year. ${ }^{53}$ These patients should receive secondary prophylaxis with antibiotics for life or until liver transplantation. ${ }^{53}$ Primary antibiotic prophylaxis should also be considered for patients with severe liver disease and ascitic fluid protein lower than $15 \mathrm{~g} / \mathrm{L}$, as these patients are at increased risk of SBP. One placebocontrolled randomized trial showed a reduction in the incidence of HRS ( $28 \%$ vs. $41 \%$ ) and an improvement in 3 month survival (94\% vs. $62 \%$ ) in this population when compared to placebo. ${ }^{54}$ In this regard, use of prophylactic antibiotics may be considered for patients with a baseline Model for End-Stage Liver Disease (MELD) score above 20. In a case controlled retrospective study, development of SBP was shown to be linearly associated with the baseline MELD score within 1 month prior to the onset of this infection. The risk was much increased with a baseline MELD score above $20 .^{55}$

\section{Management}

General management of AKI in cirrhosis involves a thorough review of the medication list and withdrawal of offending medications, volume expansion in patients with suspected hypovolemia, and prompt recognition and treatment of bacterial infection. $3,14,26$ Prerenal AKI is primarily managed by volume expansion and withholding offending medications, specifically diuretics and lactulose (in the setting of severe diarrhea). In patients with hypoalbuminemia, albumin is used for volume expansion at a dose of $1 \mathrm{~g} / \mathrm{kg}$ body weight per day to a maximum of $100 \mathrm{~g}$ albumin/day. ${ }^{14}$ While diuretics are withheld, symptomatic ascites may be managed with large volume paracentesis with albumin supplementation. ${ }^{56}$ Unrecognized AKI, severe hypovolemia, and sepsis may result in intrarenal injury due to ATN. Patients with intrarenal injury may require hemodialysis for volume overload, hyperkalemia, symptomatic uremia, or metabolic acidosis. ${ }^{3,26}$ For patients with intrinsic kidney injury secondary to hepatitis $C$ related glomerulonephropathies, antiviral treatment may result in improved renal function. ${ }^{39}$

\section{Treatment of HRS}

As alluded to earlier in this review, the diagnosis of HRS is essentially one of exclusion. In routine practice, patients are treated for HRS if a) the renal function does not resolve or improve with fluid replacement and holding medications such as diuretics and nephrotoxic medications, b) infections and sepsis are adequately treated or excluded, and c) urine sediment is normal and bland. Given that the renal dysfunction in HRS is a functional issue due to hemodynamic changes in cirrhosis, the definitive therapy for HRS is liver transplantation. ${ }^{14}$ The rationale for using vasoconstrictors for the treatment of HRS is reversal of splanchnic vasodilation and squeezing blood into the systemic circulation, thus increasing the effective arterial blood and renal blood flow. The improvement in renal perfusion results in decreased activation of renal vasoconstrictor systems and thus improved GFR. ${ }^{14}$

Terlipressin, a vasopressin analog that stimulates V1 receptors on vascular smooth muscle cells and causes both systemic and splanchnic vasoconstriction, has been used for the treatment of HRS. ${ }^{57}$ In a systematic review and 
meta-analysis of eight randomized studies on 377 patients, terlipressin reduced all-cause mortality by $15 \%$, with reduction in mortality due to HRS by $9 \%$. The authors concluded that terlipressin has survival benefits up to 3 months but only when HRS is a cause of mortality. ${ }^{58}$ In another study on 18 HRS patients, the response rate was $67 \%$ and six nonresponders were all patients with acute on chronic liver failure. ${ }^{59}$ In a meta-analysis examining the use of terlipressin for treatment of type 1 and 2 HRS, an overall improvement in short term mortality and renal function was seen in the group of patients who received terlipressin with or without albumin compared to no treatment or albumin alone. ${ }^{57}$ Continuous outpatient infusion of terlipressin has been shown to be an option as a bridge to liver transplantation. 60

In one randomized controlled study involving 46 patients comparing norepinephrine and terlipressin ( $n=23$ each), HRS was reversed in $74 \%$ cases in both groups. However, HRS recurred in $2 / 3$ of patients. ${ }^{61}$ In a systematic review and meta-analysis of four randomized studies on 154 patients comparing norepinephrine and terlipressin, there was no difference on reversal of HRS, recurrence of HRS, and 30 day mortality. However, the odds of side effects were reduced by $64 \%$ with norepinephrine, 0.36 [0.15-0.83] compared to terlipressin. ${ }^{62}$ The major limitation of norepinephrine is the need for continuous infusion with intensive care monitoring. ${ }^{14}$ Midodrine is an oral alpha- 1 adrenergic agonist that can be used for outpatients and for patients on the regular medical wards. Oral midodrine in combination with subcutaneous octreotide, a somatostatin analog, is successful in reversing HRS and improving short term mortality. ${ }^{63-65}$ Octreotide potentiates the effects of midodrine by decreasing secretion of glucagon, which plays a role in splanchnic vasodilation. ${ }^{66}$ In another study, 23 patients with HRS were randomized to receive midodrine and octreotide $(n=12)$ or epinephrine $(n=11)$. There were no differences in the efficacy, safety, and recurrence of HRS between these two drugs. ${ }^{67}$ In a randomized controlled study comparing midodrine and octreotide plus albumin to terlipressin infusion plus albumin, the rates of reversal of HRS were $70 \%$ and $29 \%$, respectively, and there was no difference in 1 and 3 month survival between the two groups. ${ }^{68}$

All of these vasoconstrictors are given in combination with intravenous albumin, which expands plasma volume and binds vasodilatory compounds, resulting in reduced vasodilation and an increase in mean arterial pressure. The benefits of albumin were demonstrated by a nonrandomized prospective study that found a significant increase in rate of reversal of HRS ( $77 \%$ vs. $25 \%$ ) and mean arterial pressure in the group of patients receiving terlipressin plus albumin compared to terlipressin alone. ${ }^{69}$ Based on this study, it is recommended to administer vasoconstrictors with albumin in the treatment of HRS. Terlipressin is not yet Food and Drug Administration (FDA) approved for use in the United States (US) but is the preferred treatment for type 1 HRS in Europe. ${ }^{14}$ Hence, alternate vasoconstrictors are used in the US pending FDA approval of terlipressin. Larger randomized studies are needed in the US to get FDA drug approval for the use of terlipressin in patients with HRS.

Although these agents are used almost exclusively for patients with type $1 \mathrm{HRS}$, a study showed that patients with cirrhosis and ascites with renal dysfunction not due to HRS may also benefit from vasoconstrictor therapy with terlipres$\sin .{ }^{70}$ While these therapies appear to be beneficial for patients with type 2 HRS, their role remains unclear. Nonrandomized studies have shown that terlipressin with or without albumin led to improved renal function in patients with type 2 HRS. ${ }^{69,71}$ Further randomized controlled trials are needed to evaluate the efficacy and long term safety of these agents in type 2 HRS.

Renal replacement therapy with dialysis may be used for HRS patients who fail medical therapy as a bridge to liver transplantation or as artificial liver support while awaiting reversal of an acute liver failure, such as acute alcoholic hepatitis. ${ }^{3,14}$ The indications for initiating dialysis in patients with HRS are the same as for patients with AKI of other etiologies and include volume overload, hyperkalemia, symptomatic uremia, pericarditis, and acidosis. The exact mode of dialysis (continuous renal replacement therapy versus intermittent hemodialysis) that is superior in patients with AKI and cirrhosis remains unclear. Risks of dialysis include hypotension, infection, and bleeding. ${ }^{72}$ Extracorporeal albumin dialysis (ECAD) has been proposed for treatment as modified activating reticular system (MARS). In a randomized study on 166 patients, survival was similar in patients receiving standard of care $(n=81)$ and patients treated with ECAD $(n=85)$. However, ECAD was superior in improving the encephalopathy, reducing bilirubin, and improving serum creatinine. ${ }^{73}$ Based on these data, ECAD may be an alternative option to bridge patients with type-1 HRS to liver transplantation.

Transjugular intrahepatic portosystemic shunt (TIPS) has also been considered for treatment of HRS and can theoretically lead to reductions in portal hypertension and thus splanchnic vasodilation. Two small case series have shown some success in improving renal function and survival in patients with type 1 HRS treated with TIPS. ${ }^{74,75}$ Furthermore, patients who undergo TIPS for refractory ascites often have improvement in renal function, although this improvement appears to be delayed. ${ }^{76}$ However, patients with advanced cirrhosis and AKI are often too sick to undergo the procedure, and TIPS carries a risk of worsened hepatic encephalopathy and bleeding. Until further evaluated with randomized controlled trials, TIPS should be considered an experimental therapy.

\section{Prognosis and relevance to liver transplantation}

The prognosis for patients with cirrhosis and AKI is very poor. ${ }^{1-3,5,32,33,39,46}$ AKI negatively impacts short- and longterm post-transplant survival. ${ }^{1-4,14,15,26,36,77}$ Also, increased duration of renal failure prior to transplant appears to be a negative predictor of post-transplant renal function. ${ }^{78} \mathrm{~A}$ study examining the effect of pretransplant renal function on survival found that an average pretransplant serum creatinine of $0.8 \mathrm{mg} / \mathrm{dL}$ was associated with a 5 year survival of $62 \%$, whereas 5 year survival in patients with pretransplant serum creatinine of $2.7 \mathrm{mg} / \mathrm{dL}$ was $42 \% .{ }^{77}$ Another study showed 5 year post-transplant survival of patients with HRS was moderately reduced compared to patients without HRS (60\% vs. $68 \%) .{ }^{79}$ Medical treatment for HRS appears to improve post-transplant outcomes and should be initiated prior to transplant. ${ }^{80}$ In regards to etiology of renal dysfunction, a retrospective study of patients undergoing OLT showed ATN as a cause of AKI to be associated with worse survival at 1 and 5 years after liver transplantation compared to patients with HRS as a cause of AKI. ${ }^{81}$ Clearly, SLK transplantation would be needed to improve post-transplant outcomes in these select patients. As for predicting prognosis, urinary and serum biomarkers will likely prove to be valuable, as previously discussed. In critically ill patients with cirrhosis and 
Table 3. Indications for simultaneous liver kidney transplant (SLK) in patients with cirrhosis and renal dysfunction

End Stage Liver Disease and one or more of the following:

- End Stage Renal Disease requiring hemodialysis

Or

- Chronic Kidney Disease with GFR $\leq 30 \mathrm{~mL} / \mathrm{min} *$ for $>3$ months

Or

- Kidney biopsy showing $>30 \%$ glomerulosclerosis or interstitial fibrosis

Or

- Acute Kidney Injury with creatinine $\geq 2.0 \mathrm{mg} / \mathrm{dL}$ and/or requiring dialysis for $\geq 8$ weeks

GFR, glomerular filtration rate; MDRD, Modification of Diet in Renal Disease.

AKI, a simple prediction model, the MBRS score (a 4 point score calculated using mean arterial pressure, serum bilirubin, and the presence of respiratory failure and sepsis), has been found to be useful in predicting in-hospital mortality. A MBRS score of $>2$ is associated with $72 \%$ in-hospital mortality and a score of 4 with $97 \%$ in-hospital mortality. ${ }^{82}$

If SLK transplantation can improve the post-transplant survival, one strategy is to use SLK in all patients with pretransplant AKI or renal insufficiency. However, the median wait time of patients on renal transplant list is about 6-8 years due to shortage of donor kidneys. As of 2008, about 40,000 to 50,000 patients are active on the transplant list and waiting for renal transplantation at any given time. ${ }^{83}$ Another option would be to use liver transplant alone for all patients with subsequent renal transplantation for patients who fail to recover renal function. However, immediate post-transplant outcomes may be inferior in patients who would have high mortality after liver transplant alone. In one study using the United Network for Organ Sharing (UNOS) database, patients listed for SLK transplantation but receiving only liver alone had much higher mortality within the first $48 \mathrm{~h}$ after transplantation compared to patients receiving SLK transplantation ( $11.4 \%$ vs. $0.5 \%, p<0.0001) .{ }^{84}$ Further, patients receiving kidney after liver have worse outcomes compared to patients receiving SLK transplantation. ${ }^{85}$

Therefore, there remains a need for accurate predictors and biomarkers for renal function recovery after liver transplantation alone. This would be helpful to better allocate donor organs and identify patients who would benefit from SLK the most. Many retrospective and national database studies have emerged with variables predictive of renal function recovery after liver transplantation alone. ${ }^{78,84,86-88}$ Based on these and many other studies, current recommendations for allocating SLK transplant among patients with cirrhosis who have renal insufficiency are: chronic kidney disease with estimated GFR (eGFR) $\leq 30 \mathrm{~mL} / \mathrm{min}$, proteinuria $>3 \mathrm{~g} / \mathrm{d},>30 \%$ glomerulosclerosis or interstitial fibrosis, and/ or AKI with requirement of dialysis and/or sustained eGFR $<25 \mathrm{~mL} / \mathrm{min}$ for $>6$ weeks (Table 3). ${ }^{89}$ These criteria are based on consensus and lack strong evidence to support. For this reason, within the past decade, the consensus criteria have been revised three times with current criteria emerging in 2012. This is often due to the inability to differentiate ATN versus HRS as the cause of AKI. Patients with HRS should recover their renal function after liver transplantation alone. Patients with intrarenal causes, such as ATN, often do not recover renal function, and these patients would need SLK transplantation. ${ }^{81}$ Further, recovery of renal function in patients with HRS after liver transplantation occurs only in about $68-75 \%$ cases, possible due to prolonged duration of renal dysfunction prior to transplant and/or the presence of preexisting chronic kidney disease. ${ }^{81,90}$

Since the MELD score was first used for liver transplantation listing in 2002, SLK transplantation has increased by over $300 \% .{ }^{89,91}$ Given our current imperfect criteria for SLK allocation and inaccurate predictors of post-transplant renal function, there remains a need for serum and/or urinary biomarkers to distinguish ATN from HRS and to predict accurately renal function recovery after liver transplantation. ${ }^{13,40}$ Over the last decade or so, many serum and/or urine biomarkers of renal tubular injury have emerged, such as NGAL, KIM-1, IL-18, IL-6, FABP-2, and ET-1. These biomarkers have shown promise in making early diagnosis of AKI and predicting reversal of AKI in surgical and intensive care settings. ${ }^{40}$ However, data are limited on their use among patients with cirrhosis and receiving liver transplantation. In a recent study, these biomarkers have shown to be accurate in distinguishing ATN from prerenal etiology of AKI. However, there was quite a bit of overlap in distinguishing HRS from ATN. ${ }^{13}$ More prospective data are needed with well-designed studies to define whether these biomarkers are able to accurately predict recovery of renal function after transplant. Making this determination will help to identify patients who would benefit from SLK most and more optimally utilize donor kidneys, which are already scarce.

\section{Conclusions}

In conclusion, AKI is a common occurrence in patients with advanced cirrhosis and is associated with high morbidity and mortality. Recognizing the common causes of AKI in cirrhosis along with early diagnosis and treatment is imperative for improving outcomes. Future research needs to be done to further elucidate the role of vasoconstrictors plus albumin in type 2 HRS and to examine the relationship between urinary biomarkers and transplant outcomes so that we may improve the allocation of donor organs to those who need them most.

\section{Conflict of interest}

None

\section{Author contributions}

Drafting of manuscript (KBR), concept and critical revision with intellectual content (AKS), providing pathology pictures (TMS).

\section{References}

[1] D'Amico G, Garcia-Tsao G, Pagliaro L. Natural history and prognostic indicators of survival in cirrhosis: a systematic review of 118 studies. J Hepatol 2006;44:217-231. doi: 10.1016/j.jhep.2005.10.013.

[2] du Cheyron D, Bouchet B, Parienti JJ, Ramakers M, Charbonneau P. The attributable mortality of acute renal failure in critically ill patients with liver cirrhosis. Intensive Care Med 2005;31:1693-1699. doi: 10.1007/s00134005-2842-7.

[3] Ginès P, Schrier RW. Renal failure in cirrhosis. N Engl J Med 2009;361:12791290. doi: 10.1056/NEJMra0809139. 
[4] Montoliu S, Ballesté B, Planas R, Alvarez MA, Rivera M, Miquel M, et al. Incidence and prognosis of different types of functional renal failure in cirrhotic patients with ascites. Clin Gastroenterol Hepatol 2010;8:616-622. quiz e80. doi: $10.1016 / \mathrm{j} . c g h .2010 .03 .029$.

[5] Wu CC, Yeung LK, Tsai WS, Tseng CF, Chu P, Huang TY, et al. Incidence and factors predictive of acute renal failure in patients with advanced liver cirrhosis. Clin Nephrol 2006;65:28-33.

[6] Terra C, Guevara M, Torre A, Gilabert R, Fernández J, Martín-Llahí M, et al. Renal failure in patients with cirrhosis and sepsis unrelated to spontaneous bacterial peritonitis: value of MELD score. Gastroenterology 2005;129: 1944-1953. doi: 10.1053/j.gastro.2005.09.024.

[7] Hampel H, Bynum GD, Zamora E, El-Serag HB. Risk factors for the development of renal dysfunction in hospitalized patients with cirrhosis. Am J Gastroenterol 2001;96:2206-2210. doi: 10.1111/j.1572-0241.2001.03958.x.

[8] Sherman DS, Fish DN, Teitelbaum I. Assessing renal function in cirrhotic patients: problems and pitfalls. Am J Kidney Dis 2003;41:269-278. doi: 10.1053/ajkd.2003.50035.

[9] Wong F, Nadim MK, Kellum JA, Salerno F, Bellomo R, Gerbes A, et al. Working Party proposal for a revised classification system of renal dysfunction in patients with cirrhosis. Gut 2011;60:702-709. doi: 10.1136/gut.2010. 236133.

[10] Caregaro L, Menon F, Angeli P, Amodio P, Merkel C, Bortoluzzi A, et al. Limitations of serum creatinine level and creatinine clearance as filtration markers in cirrhosis. Arch Intern Med 1994;154:201-205. doi: 10.1001/ archinte.1994.00420020117013.

[11] Spencer K. Analytical reviews in clinical biochemistry: the estimation of creatinine. Ann Clin Biochem 1986;23:1-25.

[12] Chawla LS, Kimmel PL. Acute kidney injury and chronic kidney disease: an integrated clinical syndrome. Kidney Int 2012;82:516-524. doi: $10.1038 / \mathrm{ki}$. 2012.208.

[13] Belcher JM, Garcia-Tsao G, Sanyal AJ, Bhogal H, Lim JK, Ansari N, et al. Association of AKI with mortality and complications in hospitalized patients with cirrhosis. Hepatology 2013;57:753-762. doi: 10.1002/hep.25735.

[14] Angeli $P$, Ginès $P$, Wong F, Bernardi $M$, Boyer TD, Gerbes A, et al. Diagnosis and management of acute kidney injury in patients with cirrhosis: Revised consensus recommendations of the International Club of Ascites. J Hepatol 2015;62:968-974. doi: 10.1016/j.jhep.2014.12.029.

[15] Russ KB, Kuo YF, Singal AK. Renal function and acute kidney injury among cirhosis patients listed for liver transplantation: a prospective study. Am J Gastroenterol 2014;109:S173.

[16] Stevens LA, Levey AS. Measured GFR as a confirmatory test for estimated GFR. J Am Soc Nephrol 2009;20:2305-2313. doi: 10.1681/ASN. 2009020171.

[17] Francoz C, Nadim MK, Baron A, Prié D, Antoine C, Belghiti J, et al. Glomerular filtration rate equations for liver-kidney transplantation in cirrhotic patients: validation of current recommendations. Hepatology 2014;59:1514-1521. doi: 10.1002/hep.26704.

[18] Martin PY, Ginès P, Schrier RW. Nitric oxide as a mediator of hemodynamic abnormalities and sodium and water retention in cirrhosis. N Engl J Med 1998;339:533-541. doi: 10.1056/NEJM199808203390807.

[19] Ros J, Clària J, To-Figueras J, Planagumà A, Cejudo-Martín P, Fernández-Varo G, et al. Endogenous cannabinoids: a new system involved in the homeostasis of arterial pressure in experimental cirrhosis in the rat. Gastroenterology 2002;122:85-93. doi: 10.1053/gast.2002.30305.

[20] Navasa M, Follo A, Filella X, Jiménez W, Francitorra A, Planas R, et al. Tumor necrosis factor and interleukin- 6 in spontaneous bacterial peritonitis in cirrhosis: relationship with the development of renal impairment and mortality. Hepatology 1998;27:1227-1232. doi: 10.1002/hep.510270507.

[21] Møller S, Henriksen JH. Cirrhotic cardiomyopathy: a pathophysiological review of circulatory dysfunction in liver disease. Heart 2002;87:9-15. doi: $10.1136 /$ heart.87.1.9.

[22] Rahman S, Mallett SV. Cirrhotic cardiomyopathy: Implications for the perioperative management of liver transplant patients. World J Hepatol 2015;7: 507-520. doi: 10.4254/wjh.v7.i3.507.

[23] Kashani A, Landaverde C, Medici V, Rossaro L. Fluid retention in cirrhosis: pathophysiology and management. QJM 2008;101:71-85. doi: 10.1093/ qjmed/hcm 121 .

[24] Stadlbauer V, Wright GA, Banaji M, Mukhopadhya A, Mookerjee RP, Moore K, et al. Relationship between activation of the sympathetic nervous system and renal blood flow autoregulation in cirrhosis. Gastroenterology 2008; 134:111-119. doi: 10.1053/j.gastro.2007.10.055.

[25] Ros J, Clària J, Jiménez W, Bosch-Marcé M, Angeli P, Arroyo V, et al. Role of nitric oxide and prostacyclin in the control of renal perfusion in experimental cirrhosis. Hepatology 1995;22:915-920.

[26] Garcia-Tsao G, Parikh CR, Viola A. Acute kidney injury in cirrhosis. Hepatology 2008;48:2064-2077. doi: 10.1002/hep.22605.

[27] Moreau R, Durand F, Poynard T, Duhamel C, Cervoni JP, Ichaï P, et al. Terlipressin in patients with cirrhosis and type 1 hepatorenal syndrome: a retrospective multicenter study. Gastroenterology 2002;122:923-930. doi: $10.1053 /$ gast.2002.32364.
[28] Soares-Weiser K, Brezis M, Tur-Kaspa R, Leibovici L. Antibiotic prophylaxis for cirrhotic patients with gastrointestinal bleeding. Cochrane Database Syst Rev 2002;CD002907. doi: 10.1002/14651858.

[29] Sherman M, Klein A. AASLD single-topic research conference on hepatocellular carcinoma: Conference proceedings. Hepatology 2004;40:1465-1473. doi: $10.1002 /$ hep.20528.

[30] Navasa M, Rodés J. Bacterial infections in cirrhosis. Liver Int 2004;24: 277-280. doi: 10.1111/j.1478-3231.2004.0934.x.

[31] McCormick PA, Donnelly C. Management of hepatorenal syndrome. Pharmacol Ther 2008;119:1-6. doi: 10.1016/j.pharmthera.2008.02.012.

[32] Jouët P, Meyrier A, Mal F, Callard P, Guettier C, Stordeur D, et al. Transjugular renal biopsy in the treatment of patients with cirrhosis and renal abnormalities. Hepatology 1996;24:1143-1147. doi: 10.1002/hep.510240527.

[33] Wadei HM, Geiger X], Cortese C, Mai ML, Kramer DJ, Rosser BG, et al. Kidney allocation to liver transplant candidates with renal failure of undetermined etiology: role of percutaneous renal biopsy. Am J Transplant 2008;8:26182626. doi: 10.1111/j.1600-6143.2008.02426.x.

[34] Tanriover B, Mejia A, Weinstein J, Foster SV, Ghalib R, Mubarak A, et al. Analysis of kidney function and biopsy results in liver failure patients with renal dysfunction: a new look to combined liver kidney allocation in the post-MELD era. Transplantation 2008;86:1548-1553. doi: 10.1097/TP. 0b013e31818b22cc.

[35] Salerno F, Gerbes A, Ginès P, Wong F, Arroyo V. Diagnosis, prevention and treatment of hepatorenal syndrome in cirrhosis. Gut 2007;56:1310-1318. doi: $10.1136 /$ gut.2006.107789.

[36] Ginès A, Escorsell A, Ginès P, Saló J, Jiménez W, Inglada L, et al. Incidence, predictive factors, and prognosis of the hepatorenal syndrome in cirrhosis with ascites. Gastroenterology 1993;105:229-236.

[37] Wong LP, Blackley MP, Andreoni KA, Chin H, Falk RJ, Klemmer PJ. Survival of liver transplant candidates with acute renal failure receiving renal replacement therapy. Kidney Int 2005;68:362-370. doi: 10.1111/j.1523-1755. 2005.00408.x

[38] Prakash J, Mahapatra AK, Ghosh B, Arora P, Jain AK. Clinical spectrum of renal disorders in patients with cirrhosis of liver. Ren Fail 2011;33:40-46. doi: 10.3109/0886022X.2010.541582.

[39] Latt N, Alachkar N, Gurakar A. Hepatitis C virus and its renal manifestations: a review and update. Gastroenterol Hepatol (N Y). 2012;8:434-445.

[40] Siew ED, Ware LB, Ikizler TA. Biological markers of acute kidney injury. J Am Soc Nephrol 2011;22:810-820. doi: 10.1681/ASN.2010080796.

[41] Fagundes C, Pépin MN, Guevara M, Barreto R, Casals G, Solà E, et al. Urinary neutrophil gelatinase-associated lipocalin as biomarker in the differential diagnosis of impairment of kidney function in cirrhosis. J Hepatol 2012;57: 267-273. doi: 10.1016/j.jhep.2012.03.015.

[42] Tsai MH, Chen YC, Yang CW, Jenq CC, Fang JT, Lien JM, et al. Acute renal failure in cirrhotic patients with severe sepsis: value of urinary interleukin18. J Gastroenterol Hepatol 2013;28:135-141. doi: 10.1111/j.1440-1746. 2012.07288.x

[43] Belcher JM, Sanyal AJ, Peixoto AJ, Perazella MA, Lim J, Thiessen-Philbrook H, et al. Kidney biomarkers and differential diagnosis of patients with cirrhosis and acute kidney injury. Hepatology 2014;60:622-632. doi: 10.1002/hep. 26980.

[44] Nürnberger W. Plasma endothelin and the hepatorenal syndrome. N Engl J Med 1993;329:63.

[45] Sharawey MA, Shawky EM, Ali LH, Mohammed AA, Hassan HA, Fouad YM. Cystatin C: a predictor of hepatorenal syndrome in patients with liver cirrhosis. Hepatol Int 2011. doi: 10.1007/s12072-011-9266-y.

[46] Ahn HS, Kim YS, Kim SG, Kim HK, Min SK, Jeong SW, et al. Cystatin C is a good predictor of hepatorenal syndrome and survival in patients with cirrhosis who have normal serum creatinine levels. Hepatogastroenterology 2012; 59:1168-1173. doi: 10.5754/hge11616.

[47] Heyman SN, Rosenberger C, Rosen S. Regional alterations in renal haemodynamics and oxygenation: a role in contrast medium-induced nephropathy. Nephrol Dial Transplant 2005;20:i6-i11. doi: 10.1093/ndt/gfh1069.

[48] Peak AS, Sheller A. Risk factors for developing gadolinium-induced nephrogenic systemic fibrosis. Ann Pharmacother 2007;41:1481-1485.

[49] Bonnel AR, Bunchorntavakul C, Reddy KR. Immune dysfunction and infections in patients with cirrhosis. Clin Gastroenterol Hepatol 2011;9:727-738. doi: 10.1016/j.cgh.2011.02.031.

[50] Fasolato S, Angeli P, Dallagnese L, Maresio G, Zola E, Mazza E, et al. Renal failure and bacterial infections in patients with cirrhosis: epidemiology and clinical features. Hepatology 2007;45:223-229. doi: 10.1002/hep.21443.

[51] Sort P, Navasa M, Arroyo V, Aldeguer X, Planas R, Ruiz-del-Arbol L, et al. Effect of intravenous albumin on renal impairment and mortality in patients with cirrhosis and spontaneous bacterial peritonitis. N Engl J Med 1999;341: 403-409. doi: 10.1056/NEJM199908053410603.

[52] Thévenot T, Bureau C, Oberti F, Anty R, Louvet A, Plessier A, et al. Effect of albumin in cirrhotic patients with infection other than spontaneous bacterial peritonitis. A randomized trial. J Hepatol 2015;62:822-830. doi: 10.1016/j. jhep.2014.11.017. 
[53] Runyon BA, AASLD. Introduction to the revised American Association for the Study of Liver Diseases Practice Guideline management of adult patients with ascites due to cirrhosis 2012. Hepatology 2013;57:1651-1653. doi: 10. 1002/hep.26359.

[54] Fernández J, Navasa M, Planas R, Montoliu S, Monfort D, Soriano G, et al. Primary prophylaxis of spontaneous bacterial peritonitis delays hepatorenal syndrome and improves survival in cirrhosis. Gastroenterology 2007;133: 818-824. doi: 10.1053/j.gastro.2007.06.065.

[55] Khan R, Jennings W, Salameh H, Russ K, Skinner MJ, Mudumbi S, et al. Mo1002 MELD score is a risk for first episode of spontaneous bacetrial peritionitis in patients with cirrhosis. Gastroenterology 2014;146:S983.

[56] Ginès A, Fernández-Esparrach G, Monescillo A, Vila C, Domènech $E$, Abecasis $\mathrm{R}$, et al. Randomized trial comparing albumin, dextran 70, and polygeline in cirrhotic patients with ascites treated by paracentesis. Gastroenterology 1996;111:1002-1010. doi: 10.1016/S0016-5085(96)70068-9.

[57] Gluud LL, Christensen K, Christensen E, Krag A. Terlipressin for hepatorenal syndrome. Cochrane Database Syst Rev 2012;9:CD005162. doi: 10. 1002/14651858.CD005162.pub3.

[58] Hiremath SB, Srinivas LD. Survival benefits of terlipressin and non-responder state in hepatorenal syndrome: a meta-analysis. Indian J Pharmacol 2013; 45:54-60. doi: 10.4103/0253-7613.106436.

[59] Rodríguez E, Elia C, Solà E, Barreto R, Graupera I, Andrealli A, et al. Terlipressin and albumin for type-1 hepatorenal syndrome associated with sepsis. J Hepatol 2014;60:955-961. doi: 10.1016/j.jhep.2013.12.032.

[60] Robertson M, Majumdar A, Garrett K, Rumler G, Gow P, Testro A. Continuous outpatient terlipressin infusion for hepatorenal syndrome as a bridge to successful liver transplantation. Hepatology 2014;60:2125-2126. doi: 10. $1002 /$ hep. 27154

[61] Ghosh S, Choudhary NS, Sharma AK, Singh B, Kumar P, Agarwal R, et al. Noradrenaline vs terlipressin in the treatment of type 2 hepatorenal syndrome: a randomized pilot study. Liver Int 2013;33:1187-1193. doi: 10.1111/liv. 12179.

[62] Nassar Junior AP, Farias AQ, D' Albuquerque LA, Carrilho FJ, Malbouisson LM. Terlipressin versus norepinephrine in the treatment of hepatorenal syndrome: a systematic review and meta-analysis. PLoS One 2014;9: e107466. doi: 10.1371/journal.pone.0107466.

[63] Esrailian E, Pantangco ER, Kyulo NL, Hu KQ, Runyon BA. Octreotide/ Midodrine therapy significantly improves renal function and 30-day survival in patients with type 1 hepatorenal syndrome. Dig Dis Sci 2007;52:742-748. doi: 10.1007/s10620-006-9312-0.

[64] Angeli P, Volpin R, Gerunda G, Craighero R, Roner P, Merenda R, et al. Reversal of type 1 hepatorenal syndrome with the administration of midodrine and octreotide. Hepatology 1999;29:1690-1697. doi: 10.1002/hep.510290629.

[65] Kalambokis G, Economou M, Fotopoulos A, Al Bokharhii J, Pappas C, Katsaraki $A$, et al. The effects of chronic treatment with octreotide versus octreotide plus midodrine on systemic hemodynamics and renal hemodynamics and function in nonazotemic cirrhotic patients with ascites. Am J Gastroenterol 2005;100:879-885. doi: 10.1111/j.1572-0241.2005.40899.x.

[66] Pomier-Layrargues G, Paquin SC, Hassoun Z, Lafortune M, Tran A. Octreotide in hepatorenal syndrome: a randomized, double-blind, placebo-controlled, crossover study. Hepatology 2003;38:238-243. doi: 10.1053/jhep.2003. 50276.

[67] Tavakkoli H, Yazdanpanah K, Mansourian M. Noradrenalin versus the combination of midodrine and octreotide in patients with hepatorenal syndrome: randomized clinical trial. Int J Prev Med 2012;3:764-769.

[68] Cavallin M, Kamath PS, Merli M, Fasolato S, Toniutto P, Salerno F, et al. Terlipressin plus albumin versus midodrine and octreotide plus albumin in the treatment of hepatorenal syndrome: A randomized trial. Hepatology 2015. doi: 10.1002/hep.27709.

[69] Ortega R, Ginès P, Uriz J, Cárdenas A, Calahorra B, De Las Heras D, et al. Terlipressin therapy with and without albumin for patients with hepatorenal syndrome: results of a prospective, nonrandomized study. Hepatology 2002; 36:941-948. doi: 10.1053/jhep.2002.35819.

[70] Krag A, Møller S, Henriksen JH, Holstein-Rathlou NH, Larsen FS, Bendtsen F. Terlipressin improves renal function in patients with cirrhosis and ascites without hepatorenal syndrome. Hepatology 2007;46:1863-1871. doi: 10. 1002/hep.21901.

[71] Alessandria C, Venon WD, Marzano A, Barletti C, Fadda M, Rizzetto M. Renal failure in cirrhotic patients: role of terlipressin in clinical approach to hepatorenal syndrome type 2. Eur J Gastroenterol Hepatol 2002;14:1363-1368.

[72] Vinsonneau C, Camus C, Combes A, Costa de Beauregard MA, Klouche K, Boulain $\mathrm{T}$, et al. Continuous venovenous haemodiafiltration versus intermit- tent haemodialysis for acute renal failure in patients with multiple-organ dysfunction syndrome: a multicentre randomised trial. Lancet 2006;368: 379-385. doi: 10.1016/S0140-6736(06)69111-3.

[73] Bañares R, Nevens F, Larsen FS, Jalan R, Albillos A, Dollinger M, et al. Extracorporeal albumin dialysis with the molecular adsorbent recirculating system in acute-on-chronic liver failure: the RELIEF trial. Hepatology 2013;57: 1153-1162. doi: 10.1002/hep.26185.

[74] Brensing KA, Textor J, Strunk H, Klehr HU, Schild H, Sauerbruch T. Transjugular intrahepatic portosystemic stent-shunt for hepatorenal syndrome. Lancet 1997;349:697-698. doi: 10.1016/S0140-6736(97)24010-9.

[75] Guevara $M$, Ginès $P$, Bandi JC, Gilabert $R$, Sort $P$, Jiménez $W$, et al. Transjugular intrahepatic portosystemic shunt in hepatorenal syndrome: effects on renal function and vasoactive systems. Hepatology $1998 ; 28: 416-422$ doi: $10.1002 /$ hep. 510280219

[76] Ochs A, Rössle M, Haag K, Hauenstein $\mathrm{KH}$, Deibert $\mathrm{P}$, Siegerstetter $\mathrm{V}$, et al. The transjugular intrahepatic portosystemic stent-shunt procedure for refractory ascites. N Engl J Med 1995;332:1192-1197. doi: 10.1056/ NEJM199505043321803.

[77] Nair S, Verma S, Thuluvath PJ. Pretransplant renal function predicts survival in patients undergoing orthotopic liver transplantation. Hepatology 2002;35: 1179-1185. doi: 10.1053/jhep.2002.33160.

[78] Campbell MS, Kotlyar DS, Brensinger CM, Lewis JD, Shetty K, Bloom RD, et al. Renal function after orthotopic liver transplantation is predicted by duration of pretransplantation creatinine elevation. Liver Transpl 2005;11: 1048-1055. doi: 10.1002/lt.20445.

[79] Gonwa TA, Klintmalm GB, Levy M, Jennings LS, Goldstein RM, Husberg BS. Impact of pretransplant renal function on survival after liver transplantation. Transplantation 1995;59:361-365.

[80] Restuccia T, Ortega R, Guevara M, Ginès P, Alessandria C, Ozdogan O, et al. Effects of treatment of hepatorenal syndrome before transplantation on posttransplantation outcome. A case-control study. J Hepatol 2004;40:140-146. doi: 10.1016/j.jhep.2003.09.019.

[81] Nadim MK, Genyk YS, Tokin C, Fieber J, Ananthapanyasut W, Ye W, et al. Impact of the etiology of acute kidney injury on outcomes following liver transplantation: acute tubular necrosis versus hepatorenal syndrome. Liver Transpl 2012;18:539-548. doi: 10.1002/It.23384.

[82] Pan $\mathrm{HC}$, Jenq CC, Tsai MH, Fan PC, Chang CH, Chang MY, et al. Risk models and scoring systems for predicting the prognosis in critically ill cirrhotic patients with acute kidney injury: a prospective validation study. PloS one 2012; 7:e51094. doi: 10.1371/journal.pone.0051094.

[83] Schold JD, Sehgal AR, Srinivas TR, Poggio ED, Navaneethan SD, Kaplan B. Marked variation of the association of ESRD duration before and after wait listing on kidney transplant outcomes. Am J Transplant 2010;10:2008-2016. doi: 10.1111/j.1600-6143.2010.03213.x.

[84] Hmoud B, Kuo YF, Wiesner RH, Singal AK. Outcomes of liver transplantation alone after listing for simultaneous kidney: comparison to simultaneous liver kidney transplantation. Transplantation 2015;99:823-828. doi: 10.1097/TP. 0000000000000438

[85] Simpson N, Cho YW, Cicciarelli JC, Selby RR, Fong TL. Comparison of renal allograft outcomes in combined liver-kidney transplantation versus subsequent kidney transplantation in liver transplant recipients: Analysis of UNOS Database. Transplantation 2006;82:1298-1303.

[86] Marik PE, Wood K, Starzl TE. The course of type 1 hepato-renal syndrome post liver transplantation. Nephrol Dial Transplant 2006;21:478-482. doi: $10.1093 / \mathrm{ndt} / \mathrm{gfi} 212$.

[87] Pawarode A, Fine DM, Thuluvath PJ. Independent risk factors and natural history of renal dysfunction in liver transplant recipients. Liver Transpl 2003;9:741-747. doi: 10.1053/jlts.2003.50113.

[88] Jeyarajah DR, McBride M, Klintmalm GB, Gonwa TA. Combined liver-kidney transplantation: what are the indications? Transplantation 1997;64:10911096.

[89] Eason JD, Gonwa TA, Davis CL, Sung RS, Gerber D, Bloom RD. Proceedings of Consensus Conference on Simultaneous Liver Kidney Transplantation (SLK). Am J Transplant 2008;8:2243-2251. doi: 10.1111/j.1600-6143.2008. 02416.x.

[90] Wong F, Leung W, Al Beshir M, Marquez M, Renner EL. Outcomes of patients with cirrhosis and hepatorenal syndrome type 1 treated with liver transplantation. Liver Transpl 2015;21:300-307. doi: 10.1002/It.24049.

[91] Singal AK, Salameh H, Kuo YF, Wiesner RH. Evolving frequency and outcomes of simultaneous liver kidney transplants based on liver disease etiology. Transplantation 2014;98:216-221. doi: 10.1097/TP.0000000000000048. 